\title{
Analysis of Controllability of Complex Networks
}

\author{
Zong-Yuan Tan ${ }^{1, \text { a Ning Cai }}{ }^{1, b^{*}} \mathrm{Ji}-$ Peng $\mathrm{Gu}^{1, \mathrm{c}}$ and Jian Zhou ${ }^{1, \mathrm{~d}}$ \\ ${ }^{1}$ College of Electrical Engineering, Northwest Minzu University, Lanzhou, China \\ ctanzongyuansmile@163.com, bcaining91@tsinghua.org.cn, ${ }^{c} 2026321998 @ q q . c o m$ \\ dzhoujianfrank@126.com \\ *The corresponding author: Cai Ning (caining91@tsinghua.org.cn)
}

Keywords: Complex networks; Controllability; Stability; Node noise

\begin{abstract}
The feature-based modeling methods of networks is sharply increasing difficulty with the complication of networks, which usually extremely enhances calculated works of modeling. It leads to the fact that one could not exactly simulates system action. In order to address this problem, a dynamical simulation model that directly form by empiricism is urgently established, which is based on compressed sensing system recognition and prediction.
\end{abstract}

\section{Introduction}

With the continuous development of society, network structures have been more and more complex and people also are still more interested in networks. Networks have played an important role in our daily life, particularly communication networks have produced a significant effect on fundamental communication. In recent years, a great many of scholars extremely focus on complex networks and also establish feasible network topology for simulating a variety of behaviors of complex networks to research different properties of networks.

Although complex networks and dynamical systems have obtained prominent achievement in reality, it is still faced with new challenge, which is that constantly increasing network topologies affect study of modeling and simulation of researcher [1,3]. Meanwhile, it is lack of various effective network models to forecast and operate the capacity of system behaviors for promoting research to move with an ideal direction. In order to overcome those new challenge, scholars industriously research network topologies and node dynamics for further facilitating the development of different disciplines. In addition, in the study modeling of complex networks, previous articles mainly build simple network space-time systems to reveal connected pattern and analysis of time series among agents for further analyze controllability of agents [4,5].

However, if the way applies to engineering practice, it is uneasy to realize dynamical capture of network systems, which will decrease the value of engineering research [6,9]. With networks and control theory gradually trending to integration, using modes of topology to simulate the internal behaviors of complex networks have been more and more maturity. Liu et al. develop a tool to deal with controllability of arbitrary size network topologies, which could use certain nodes as main leaders to systematically control arbitrary followers for reaching full control of systems. Hence, those methods could motivate scholars using simple topology to simulate complex large-scale networks $[10,11]$.

\section{Time-Delay Pinning Control of Complex Networks}

Consider once again a diffusively and linearly coupled network, the equation can be formulated as follows:

$$
\dot{x}_{i}=f\left(x_{i}\right)-c \sum_{j=1}^{N} a_{i j} \Gamma x_{j}
$$

Where all notations are defined as before. The objective is to stabilize the network into the 
equilibrium of the complex network, in the sense that

$$
x_{1}(t) \rightarrow x_{2}(t) \rightarrow \cdots \rightarrow x_{N}(t) \rightarrow \bar{x} \text {, as } t \rightarrow \infty
$$

Where for simplicity it is assumed that $f(0)=0$, so $\bar{x}=0$. Without loss of generality, suppose that nodes $1,2, \ldots, l$, are selected for pinning control. Hence, the controlled network can be written as the following formulation.

$$
\left\{\begin{array}{c}
\dot{x}_{i}=f\left(x_{i}\right)-c \sum_{j=1}^{N} a_{i j} \Gamma x_{j}+u_{i}, i=1,2, \cdots, l \\
\dot{x}_{i}=f\left(x_{i}\right)-c \sum_{j=1}^{N} a_{i j} \Gamma x_{j}, i=l+1, l+2, \cdots, N
\end{array}\right.
$$

Stability also is a significant research direction of network topologies and combine controllability, which form theoretical foundation of most system analysis and synthetical problems [12]. Therefore, the controllability and stability analysis of agents of network topologies could contribute to further research the properties of network topology. For instance, a star-shaped network of $N$ nodes has a Laplacian matrix:

$$
L_{s c}=\left[\begin{array}{ccccc}
N-1 & -1 & -1 & \cdots & -1 \\
-1 & 1 & 0 & \cdots & 0 \\
\cdots & & & & \cdots \\
-1 & 0 & \cdots & 1 & 0 \\
-1 & 0 & 0 & \cdots & -1
\end{array}\right]
$$

Its smallest nonzero eigenvalue $\lambda_{S C 2}=1$, which is complex degree of network size. Therefore, the synchronizability of a star-shaped network is independent of the network size.

In particular, for type-II networks, for a nearest-neighbor coupled network of $N$ nodes with even degree $K$, its eigenratio satisfies are as follows:

$$
\frac{\lambda_{N n c}}{\lambda_{2 n c}} \approx \frac{(3 \pi+2) N^{2}}{2 \pi^{2}(K+1)(K+2)}
$$

And

$$
\frac{d \theta_{i}}{d t}=\omega_{i}+\lambda \sum_{j=1}^{N} a_{i j} \sin \left(\theta_{j}-\theta_{i}\right)
$$

In the above equation, we use the value of complex network node degrees and produced a controllable matrix for a random network. With networks and control theory gradually trending to integration, using modes of topology to simulate the internal behaviors of complex networks have been more and more maturity [13].

\section{Simulation and Analysis}

In this section, we endeavor to simulate the relation of the controllability and stability of complex and the number of node, subsequently, according to the actual data, we formulate a purpose equation. The modern control theory and modern engineering have already into the field of scientometrics and complex networks, especially in epidemic spreading, as clearly shown in Fig. 1. 


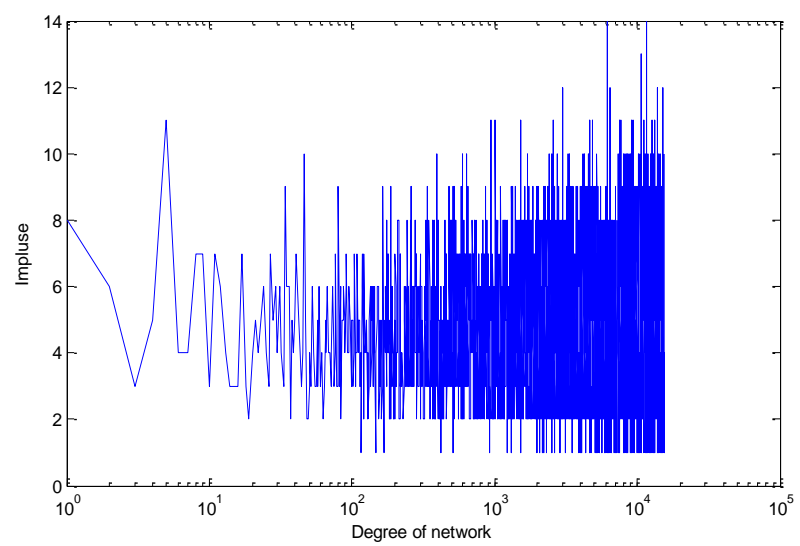

Figure 1. Relation of degree of network and impulse

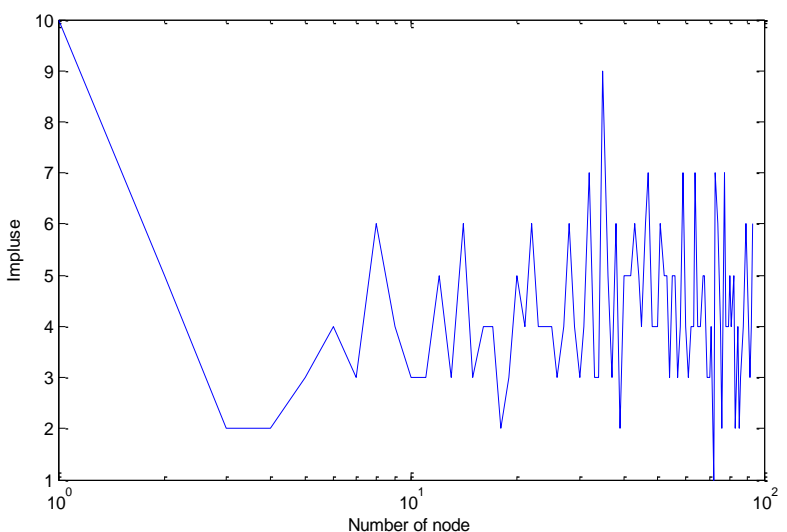

Figure 2 . Relation of number of node and impluse

Neurons are widely accepted to be organized into networks, and neuronal networks exchange information through electrical and chemical synapses. Increasing evidences indicate that astrocytes are also organized into networks, and astrocyte networks are interconnected through gap junction channels. Only a fraction of neurons are connected to autapse not all.

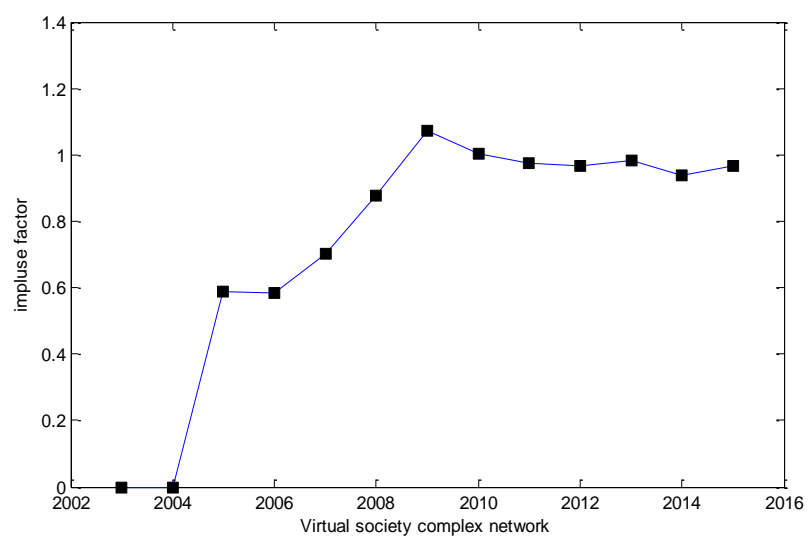

Figure 3 . Relation of virtual society complex network and impluse factor

Cognitive non-linear complex is considered capable of information processing in the social computing system. Theoretical models have been set up degrees from microcosmic, mesoscopic, up to macroscopic scale, so that the generation of cognition and potential mechanisms underlying neural information processing could be discerned. 


\section{Conclusions and Discussion}

Since about ten years ago, the controllability problems of the large-scale systems of dynamical networks have attracted a great number of scholars that are from control and physics domain, which could be expected to give rise to attention of extensive disciplines. Tanner firstly research single leader to determine the controllability problems of a system. Next, Cai et al. addressed the controllability problems of high-order systems and also proposed an improvement scheme for controllability. At the same time, Liu et al. dealt with the controllability problems of complex networks and quantitatively analyzed the controllability of networks by single index. Finally, it is in the aspect of network protocols that $\mathrm{Ji}$ et al. strived to combine the influence of controllability to further address interactive protocols among networked agents.

We endeavor to explore the controllability problems of any given network topology and also combine the intelligent character of dynamic nodes to effectively research the interaction among dynamic networks. Namely, it further analyzes the output of any other agents by the given external input, which could analyze the relation between leaders and followers to determine the controllability among agents. By this way, we research possible ways that are from qualitative to quantitative to analyze controllability of complex networks.

\section{References}

[1] J. X. Xi, M. He, H. Liu and J. F. Zheng: J Franklin Inst, Vol. 353 (2016) No.16, pp.4074-4090.

[2] N. Cai and M. J. Khan: Int. J. Control Automat. Syst., Vol. 15 (2017) No.1, pp.169-177.

[3] N. Cai, J. W. Cao, H. Y. Ma and C. X. Wang: Arab J Sci Eng, Vol. 39 (2014) No.3, pp. 2427-2434.

[4] Y. Y. Liu, J. J. Slotine and A. L. Barabási: Nature, Vol. 473 (2011) No.7346, pp.167-173.

[5] H. G. Tanner: Proc. 43rd IEEE Conf. Decision and Control (Bahamas December 14-17, 2004), Vol. 3, pp.2467-2472.

[6] N. Cai, M. He, Q. Wu and M. J. Khan: J. Syst. Sci. Complexity, DOI: 10.1007/s11424-017-6273-7.

[7] N. Cai, J. X. Xi and Y. S. Zhong et al.: Int. J. Innov. Comput. I., Vol. 8 (2012) No.5a, pp.3315-3328.

[8] Y. Q. Guan, Z. J. Ji, L. Zhang and L. Wang: Int. J. Robust Nonlin. Control, DOI: 10.1002/rnc.3798.

[9] Z. J. Ji, H. Lin and H. S. Yu: IEEE Trans. Automat. Control, Vol. 60 (2015) No.3, pp.781-786.

[10] N. Cai, J. W. Cao and M. J. Khan: Int. J. Control Automat. Syst., Vol. 12 (2014) No.6, pp.1366-1371.

[11] Z. J. Ji and H. S. Yu: IEEE Trans. Cybernet., DOI: 10.1109/TCYB.2016.2549034.

[12] N. Cai, C. Diao and M. J. Khan: Complexity, 4978613, 2017.

[13] B. Liu, T. G. Chu and L. Wang et al.: IEEE Trans. Autom. Control, Vol. 53 (2008) No.4, pp.1009-1013. 